\title{
Physician Wellness Measures and Clinical Performance on a Critically Ill Simulated Patient: Does a Lack of Well-Being Impact Patient Care?
}

\author{
Cynthia Peng ${ }^{1}$, Linn Lung ${ }^{2}$, Madeline Grade ${ }^{3}$, Nelson Wong ${ }^{4,1,5}$, Rebecca Smith-Coggins ${ }^{1}$ \\ 1. Emergency Medicine, Stanford School of Medicine, Palo Alto, USA 2. Emergency Medicine, Kaiser San Rafael \\ Hospital, San Rafael, USA 3. Emergency Department, University of California San Francisco, San Francisco, USA 4. \\ Emergency Medicine, VA Palo Alto Health Care System, Palo Alto, USA 5. Simulation, SimLEARN, Palo Alto, USA
}

Corresponding author: Cynthia Peng, cpengr@gmail.com

\section{Abstract}

\section{Background/Objective:}

Burnout is common among resident physicians, which has the potential to translate into diagnostic and management errors. Our study investigates the relationship between sleepiness, depression, anxiety, burnout, and lack of professional fulfillment with clinical performance during a critically ill patient simulation.

\section{Methods/Approach:}

Emergency medicine residents were recruited to participate in a high-fidelity simulation case of a critically ill patient. A survey with validated wellbeing measures (National Institutes of Health Patient-Reported Outcomes Measurement Information System (NIH PROMIS), Linzer burnout measure, and professional fulfillment index) was administered prior to the simulation. Each encounter was video-recorded and analyzed by two blinded raters based on a binary critical-actions checklist. Time-to-intubation, management errors, and misdiagnosis rates were assessed.

\section{Results:}

Twenty residents participated, with most subjects endorsing sleepiness (70\%) and less than half reporting depression (40\%) and anxiety (45\%). Burnout was identified to be in $50 \%$ of participants by the Linzer measure and $85 \%$ by the professional fulfillment index. No significant difference was found between mean performance scores in sleepy, depressed, and anxious cohorts in comparison to groups without those symptoms. Similarly, burnout and professional fulfillment did not yield any significant difference, nor did comparisons with time to intubation, management errors, and frequency of misdiagnosis.

\section{Conclusion:}

Received 04/23/2021 Review began 05/11/2021 Review ended 06/16/2021 Published 07/13/2021

\section{() Copyright 2021}

Peng et al. This is an open access article distributed under the terms of the Creative Commons Attribution License CC-BY 4.0., which permits unrestricted use, distribution, and reproduction in any medium, provided the original author and source are credited.
Resident burnout, depression, anxiety, sleepiness, and lack of professional fulfillment did not appear to have a measurable impact on clinical performance in managing a critically ill patient. There is no evidence from this study that the lack of resident physician well-being adversely impacts patient care by increasing errors in management or misdiagnoses during this high-fidelity simulation.

Categories: Emergency Medicine, Medical Education, Medical Simulation

Keywords: medical education, critical care simulation, resident physician, clinical performance, emergency medicine

\section{Introduction}

Burnout has been defined by Dr. Christina Maslach as having three key components: 1) a feeling of overwhelming exhaustion, 2) cynicism and detachment from the job, 3) and a sense of ineffectiveness and lack of accomplishment [1]. These symptoms often begin manifesting early in medical learners during medical school with increasing depersonalization and emotional exhaustion [2,3]. Burnout, as well, is cited as a commonplace syndrome among the United States (US) physicians, with an alarming prevalence of 40$50 \%$ in physicians at all career stages [4]. Threats to well-being include factors such as lack of sleep and the co-existence of depression and anxiety [5].

The complex interplay of these entities in trainees may have an impact on clinical performance. In Fahrenkopf et al., significantly more medical errors were made by residents who met the criteria for depression but not burnout [2]. Similarly, in simulated medical scenarios, lower cumulative performance scores on high-fidelity simulation scenarios have been associated with emergency medical trainees exhibiting signs of burnout [6]. 
The margin for medical errors in high-stakes medical situations, such as that of a critically ill patient, is low. Misdiagnosis, management mistakes, and delay in action can lead to devastating consequences. There has been limited investigation into the relationship between quality of care delivered and metrics of wellness as measured by the physicians in charge of the resuscitation.

Our study attempts to investigate the correlation between wellness deficits reported by emergency medicine residents in sleepiness, depression, anxiety, burnout, and lack of professional fulfillment and their subsequent clinical performance on a simulated critically ill patient encounter.

\section{Materials And Methods}

This was a cross-sectional, single-center prospective study in a Level 1 Trauma Center. We recruited a convenience sample of emergency medicine residents, post-graduate year (PGY) one through four, to participate from Jan 2018-March 2019. The study was approved by the Stanford Institutional Review Board (IRB).

All subjects completed a baseline survey, which included wellness assessment instruments as well as basic demographic information. Items included the short form version for National Institutes of Health PatientReported Outcomes Measurement Information System (NIH PROMIS) measures of depression, sleep-related impairment, and anxiety [7]. Burnout was measured with a psychometrically validated single-item question from the Maslach Burnout Inventory and the Professional Fulfillment Index (PFI) scale [8,9].

Each subject participated in a high-fidelity simulation case involving a critically ill patient: a patient presenting to the Emergency Department (ED) from a house fire with carbon monoxide and cyanide poisoning, which required rapid sequence intubation and initiation of other therapies. A binary criticalactions checklist was created based on the expert consensus of three practicing emergency physicians. An audiovisual recording was collected of each simulation session. The simulation session was run by two simulation-trained emergency medicine faculty.

Two independent raters with clinical medical backgrounds and simulation training were blinded to the identity and experience level of the participants. They rated each trainee's simulation performance based on an itemized checklist (maximum score of 21). Discrepant performance items were resolved by a third reviewer. Additional data measured included time-to-intubation, management error, and misdiagnosis rates. Management error was defined as missing greater than or equal to one critical intervention and/or treatment. Misdiagnosis was broadly defined as missing or having the incorrect final diagnosis.

Statistical analysis on survey results and simulation performance was performed using SPSS version 25 (IBM Inc., Armonk, USA). Descriptive statistics were analyzed, and the normality of the dependent variables was assessed using the Shapiro-Wilk test. Simulation scores and time to intubation were compared to the NIH PROMIS measures using a parametric independent $t$-test with a significance level of 0.05 . For the other well-being measures that did not have normally distributed data, the Kruskal-Wallis and Wilcoxon rank-sum test was utilized.

\section{Results}

Twenty residents were recruited and participated in the study (Table 1). Of the participants, 68\% were male, and $32 \%$ were female. $45 \%$ representing the PGY2 class and 55\% PGY3 class. $63 \%$ of the participants were married or in domestic partnerships, and $75 \%$ reported Caucasian ethnicity. 


\section{Cureus}

Characteristic

Frequency

Age $^{*}$

$<$ or $=30$ years old

$10(56 \%)$

$>30$ years old

8 (44\%)

Training Year

PGY2

$9(45 \%)$

PGY3

11 (55\%)

Gender*

Female

$6(32 \%)$

Male

$13(68 \%)$

Ethnicity

Caucasian

15 (75\%)

Other

$5(25 \%)$

Family status*

Single

$7(37 \%)$

Married or domestic partnership

$12(63 \%)$

Children

$4(21 \%)$

\section{TABLE 1: Descriptive characteristics of participants}

*incomplete entries were excluded from designated categories

The majority of participants $(70 \%, \mathrm{n}=14)$ endorsed sleepiness, and $40 \%$ screened positive for depression

$(n=8)$ and $45 \%$ for anxiety $(n=9)$ (Table 2$)$. There were no differences in burnout measures between male and female groups. Burnout was identified in $50 \%$ of participants $(n=10)$. 


\section{Cureus}

\section{Sleepiness}

Y

Depression

Anxiety

$12(60 \%)$

Anxiety

\section{Burnout}

Professional Fulfillment

Y

N

$8(40 \%)$

Professional Fulfillment Burnout

\section{TABLE 2: Wellness metric measures and participant responses}

No significant difference was found in mean simulation performance scores between the sleepy versus not sleepy cohorts (15 vs. 15.5). This finding was congruent for depression and anxiety measures as well (Table 3). Similarly, burnout versus no burnout groups and professionally fulfilled versus not fulfilled groups did not yield any significant difference in simulation performance scores. There was also no significant difference in time to intubation, management errors, and frequency of misdiagnosis between all wellness measure groups. 


\section{Cureus}

\begin{tabular}{|c|c|c|c|c|}
\hline & Simulation Score & Time to Intubation (seconds) & $>1$ Management error & $>1$ Misdiagnosis \\
\hline \multicolumn{5}{|c|}{ Sleepiness } \\
\hline $\mathrm{Y}$ & $15 \pm 2.5$ & $294.9 \pm 169.9$ & $85.7 \%$ & $55.6 \%$ \\
\hline $\mathrm{N}$ & $15.5 \pm 1.1$ & $274.8 \pm 57.3$ & $66.7 \%$ & $33.3 \%$ \\
\hline \multicolumn{5}{|c|}{ Depression } \\
\hline $\mathrm{Y}$ & $15 \pm 2.9$ & $250.9 \pm 108.1$ & $75.0 \%$ & $25.0 \%$ \\
\hline $\mathrm{N}$ & $15.3 \pm 1.5$ & $314.3 \pm 163.0$ & $83.3 \%$ & $41.7 \%$ \\
\hline \multicolumn{5}{|c|}{ Anxiety } \\
\hline Y & $15.6 \pm 2.4$ & $288.1 \pm 91.3$ & $77.8 \%$ & $22.2 \%$ \\
\hline $\mathrm{N}$ & $14.8 \pm 1.9$ & $289.5 \pm 180.7$ & $81.8 \%$ & $45.5 \%$ \\
\hline \multicolumn{5}{|c|}{ Burnout } \\
\hline $\mathrm{Y}$ & $16 \pm 1.8$ & $331.3 \pm 171.4$ & $80.0 \%$ & $20.0 \%$ \\
\hline $\mathrm{N}$ & $14.3 \pm 2.2$ & $246.5 \pm 101.6$ & $80.0 \%$ & $50.0 \%$ \\
\hline \multicolumn{5}{|c|}{ Professional Fulfillment } \\
\hline Y & $14.8 \pm 2.4$ & $263.8 \pm 103.5$ & $75.0 \%$ & $41.7 \%$ \\
\hline $\mathrm{N}$ & $15.6 \pm 1.6$ & $326.5 \pm 191.5$ & $87.5 \%$ & $25.0 \%$ \\
\hline \multicolumn{5}{|c|}{ Professional rulfillment Burnout } \\
\hline $\mathrm{Y}$ & $15 \pm 2.2$ & $289.7 \pm 156.5$ & $82.4 \%$ & $35.3 \%$ \\
\hline $\mathrm{N}$ & $16 \pm 1$ & $284.3 \pm 25.4$ & $66.7 \%$ & $33.3 \%$ \\
\hline
\end{tabular}

TABLE 3: Composite simulation performance measures versus wellness measures

Subanalysis revealed no difference in PGY2 and PGY3 simulation scores (15.5 vs. 14.8), time to intubation (241 seconds vs. 327 seconds), management error ( $77.8 \%$ vs. $81.8 \%$ ), or frequency of misdiagnosis (33.3\% vs. 36.4\%). Below-average performers (under a score of 15 ) did not display more signs of sleepiness, depression, anxiety, or burnout compared to peers that performed better.

\section{Discussion}

In this simulation case of a critically ill patient, emergency medicine resident performance was not significantly impacted by their self-reported sleepiness, depression, anxiety, burnout, or professional fulfillment. Importantly, there were no significant delays in intubation, increase in management errors or additional critical misdiagnoses. With simulation performance as a proxy for clinical performance, our findings suggest that providers may be able to perform adequately when caring for individual critically ill patients even in the context of scoring lower measures on various wellness metrics.

Overall, the rates of depression, anxiety, and burnout in this study cohort were fairly similar to metaanalysis prevalence data among resident physicians. Prevalence of all levels of depressive symptoms among residents broadly ranges from $20.9 \%$ to $43.2 \%$ [10].

The cognitive load from taking care of critically ill patients may be higher than for lower acuity patient care. However, in the face of a rapidly decompensating patient, it may require the full engagement of the physician to stabilize the crisis. Our study suggests that the lack of wellness may not be evident in this scenario and does not translate to negligent care. This is concordant with prior limited work in this area [6]. Further studies will be needed to investigate if this is also similar in settings of routine patient care or in the care of multiple critical patients.

Our study highlights the adaptive nature of our medical learners. This perhaps showcases the self-selection into medicine of individuals with underlying resilient traits. The adversities and stressors encountered during years of medical training may promote the development of resilience [11]. This may allow trainees to retain high performance even when experiencing symptoms of depression, anxiety, sleepiness, and 
burnout. Interestingly, learners who had below-average performances did not report worsened wellness across our metrics. This further supports our findings that lack of well-being is not necessarily related to performance.

\section{Conclusions}

In conclusion, resident burnout, depression, anxiety, sleepiness, and lack of professional fulfillment did not appear to have a measurable effect on overall clinical performance on this high-fidelity simulation of a critically ill patient.

Our study was not without limitations. We had a strictly defined cohort of emergency medicine residents from a single center. In addition, the sensitivity of the measures of performance within our specific simulation was not previously validated in the literature and is difficult to generalize to the care of all critically ill patients. In addition, certain wellness metrics, such as the NIH PROMIS measures, were developed based on the general population and not for our specific cohort. Our study invites future studies to further delineate the relationship between wellness and care of critically ill patients with a larger sample of learners or more complex simulations.

Further discrimination between determinants of wellness and their effect on clinical performance outside of the simulated setting is warranted. In addition, the direct implications of this on patient care outcomes is a fertile area for future investigations.

\section{Additional Information \\ Disclosures}

Human subjects: Consent was obtained or waived by all participants in this study. Stanford IRB issued approval IRB-41325. Animal subjects: All authors have confirmed that this study did not involve animal subjects or tissue. Conflicts of interest: In compliance with the ICMJE uniform disclosure form, all authors declare the following: Payment/services info: All authors have declared that no financial support was received from any organization for the submitted work. Financial relationships: All authors have declared that they have no financial relationships at present or within the previous three years with any organizations that might have an interest in the submitted work. Other relationships: All authors have declared that there are no other relationships or activities that could appear to have influenced the submitted work.

\section{References}

1. Maslach C, Leiter MP: New insights into burnout and health care: strategies for improving civility and alleviating burnout. Med Teach. 2017, 39:160-3. 10.1080/0142159X.2016.1248918

2. Hansell MW, Ungerleider RM, Brooks CA, Knudson MP, Kirk JK, Ungerleider JD: Temporal trends in medical student burnout. Fam Med. 2019, 51:399-404. 10.22454/FamMed.2019.270753

3. Dyrbye LN, Moutier C, Durning SJ, et al.: The problems program directors inherit: medical student distress at the time of graduation. Med Teach. 2011, 33:756-8. 10.3109/0142159X.2011.577468

4. Dyrbye LN, Varkey P, Boone SL, Satele DV, Sloan JA, Shanafelt TD: Physician satisfaction and burnout at different career stages. Mayo Clin Proc. 2013, 88:1358-67.

5. Feng S, Yi JS, Deitz G, Ding L, Van Gelder RN, Menda S: Relationships between sleep, activity, and burnout in ophthalmology residents. J Surg Educ. 2021, 78:1035-40. 10.1016/j.jsurg.2020.09.003

6. Lu DW, Dresden SM, Mark Courtney D, Salzman DH: An investigation of the relationship between emergency medicine trainee burnout and clinical performance in a high-fidelity simulation environment. AEM Educ Train. 2017, 1:55-9. 10.1002/aet2.10004

7. Cella D, Choi SW, Condon DM, et al.: PROMIS ${ }^{\circledR}$ adult health profiles: efficient short-form measures of seven health domains. Value Health. 2019, 22:537-44. 10.1016/j.jval.2019.02.004

8. Dolan ED, Mohr D, Lempa M, Joos S, Fihn SD, Nelson KM, Helfrich CD: Using a single item to measure burnout in primary care staff: a psychometric evaluation. J Gen Intern Med. 2015, 30:582-7. 10.1007/s11606014-3112-6

9. Trockel M, Bohman B, Lesure E, Hamidi MS, Welle D, Roberts L, Shanafelt T: A brief instrument to assess both burnout and professional fulfillment in physicians: reliability and validity, including correlation with self-reported medical errors, in a sample of resident and practicing physicians. Acad Psychiatry. 2018, 42:11-24. 10.1007/s40596-017-0849-3

10. Mata DA, Ramos MA, Bansal N, Khan R, Guille C, Di Angelantonio E, Sen S: Prevalence of depression and depressive symptoms among resident physicians: a systematic review and meta-analysis. JAMA. 2015, 314:2373-83. 10.1001/jama.2015.15845

11. Winkel AF, Honart AW, Robinson A, Jones AA, Squires A: Thriving in scrubs: a qualitative study of resident resilience. Reprod Health. 2018, 15:53. 10.1186/s12978-018-0489-4 